\title{
Research on "Cylinder-Four-Beam" Microstructure Improvement of MEMS Bionic Vector Hydrophone
}

\author{
Zeming Jian $\mathbb{D},{ }^{1}$ Yifan Huang $\mathbb{D},{ }^{1}$ Lei Nie, ${ }^{1}$ Mengran Liu $\mathbb{D},{ }^{1}$ and Guojun Zhang ${ }^{2}$ \\ ${ }^{1}$ Hubei Key Laboratory of Modern Manufacturing Quantity Engineering, School of Mechanical Engineering, Hubei University \\ of Technology, Wuhan, Hubei 430068, China \\ ${ }^{2}$ Key Laboratory of Instrumentation Science \& Dynamic Measurement, Ministry of Education, North University of China, \\ Taiyuan 030051, China
}

Correspondence should be addressed to Mengran Liu; liumengran1991@163.com

Received 8 January 2021; Accepted 11 May 2021; Published 9 June 2021

Academic Editor: De-Zhi Wu

Copyright (c) 2021 Zeming Jian et al. This is an open access article distributed under the Creative Commons Attribution License, which permits unrestricted use, distribution, and reproduction in any medium, provided the original work is properly cited.

\begin{abstract}
The existing MEMS bionic vector hydrophone has the problems of low-sensitivity and narrow-working band, and the sensitivity and working bandwidth cannot be improved simultaneously by changing the single microstructural parameter. In this paper, the MEMS bionic vector hydrophone microstructural parameters (length, width and height of cantilever, side length of the center block, height and radius of the rigid cylinder) have been optimized simultaneously to obtain a higher sensitivity at the almost same working bandwidth. Firstly, through the mechanical analysis of the microstructure, the objective function and feasible region are established to optimize the parameters of the microstructure, and a set of optimized parameters is obtained. Secondly, the optimized structure is verified by ANSYS simulation, and then, the optimized four-beam structure is fabricated by the MEMS manufacturing technology. Finally, these two kinds of hydrophones (the previous one and the optimized one) are produced, and their performance tests are carried out. The testing results show that the performances of the optimized hydrophone have been greatly improved, exhibiting a receiving sensitivity of $-181.2 \mathrm{~dB} @ 1 \mathrm{kHz}$ (increasing by $6.5 \mathrm{~dB}, 0 \mathrm{~dB}$ reference $1 \mathrm{~V} / \mu \mathrm{Pa}$ ), the frequency response ranging from $20 \mathrm{~Hz}$ to $1 \mathrm{kHz}$ which is the same working bandwidth as before, and a good dipole directivity. The optimization researches in this paper provide a method and idea for the performance improvement of the following MEMS vector hydrophone.
\end{abstract}

\section{Introduction}

With the continuous consumption of land resources, the huge resources in the deep ocean will become precious and provide the abundant substances that human beings need to live by including the energy, mine, and biology $[1,2]$. Sonar system is the key equipment to obtain information from the ocean. And vector hydrophone as an important part of the sonar detection devices can measure vibrating acceleration of water particle and has the advantage of noise cancellation due to its directional pattern [3-6]. Faced with the increasingly serious underwater security situation, it urgently needs to adopt new technologies and methods to meet the requirements of engineering application. MEMS technology, due to the characteristic of $3 \mathrm{M}$ (miniaturization, multiplicity and microelectronics), shows great vitality [7-10]. To this end, scholars expect to resolve current problems of large volume, poor consistency, and high cost through MEMS technology.

MEMS bionic vector hydrophone based on the bionic principle, piezoresistive principle, and MEMS technology, invented by the North University of China in 2007, is shown in Figure 1 [11], which has advantages of small volume, good consistency, and wonderful low frequency effect $[11,12]$.

However, the hydrophone has the problems of low sensitivity and narrow working bandwidth $[13,14]$. In recent years, a lot of efforts have been put into the MEMS vector hydrophone to improve its sensitivity. The "Lollipopshaped" structure MEMS vector hydrophone was produced by Liu et.al [15], and a stress centralized structure MEMS 


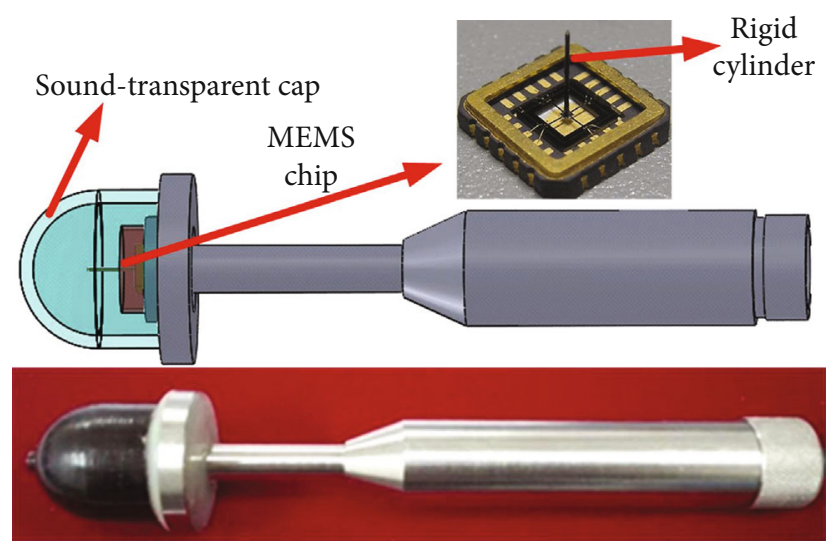

FIgURE 1: MEMS bionic vector hydrophone.

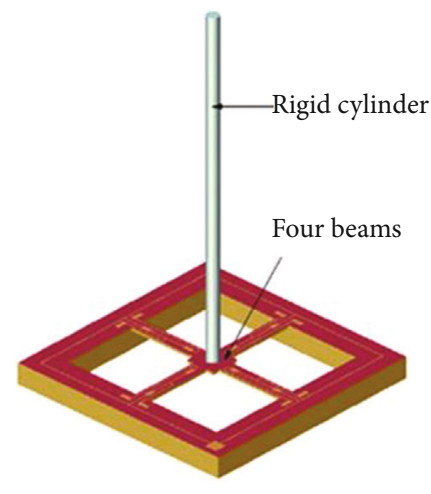

(a) The three-dimensional model

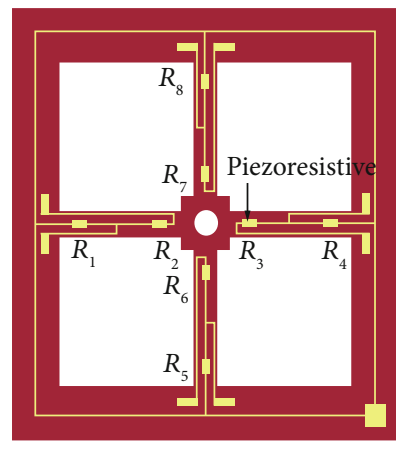

(b) The two-dimensional plan

Figure 2: The view of the hydrophone microstructure.

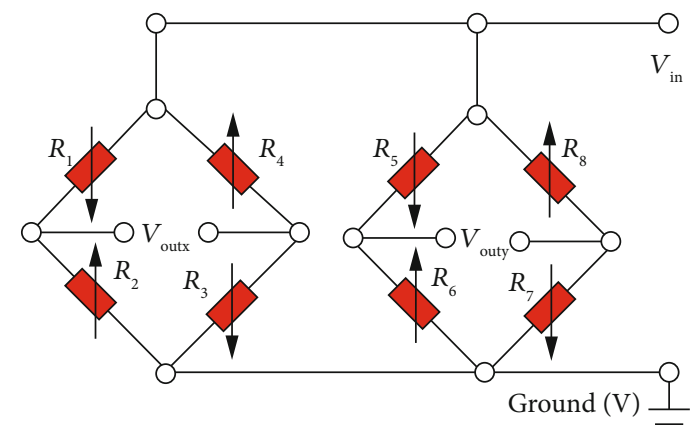

Figure 3: Diagram of Wheatstone bridge.

vector hydrophone was presented by Zhang et al. [16]. Although these two structures, respectively, improved the sensitivity of the hydrophones, it greatly reduced their working bandwidth. The sensitivity of the hydrophone has been also improved by replacing the previous cylinder structure with five different new structures, which are, respectively, the cup-shaped MEMS vector hydrophone designed by $\mathrm{Xu}$ et al. [17], the two-component cilia cylinder MEMS vector hydrophone proposed by $\mathrm{Xu}$ et al. [18], the cilia cluster MEMS vector hydrophone developed by Zhang et al. [19], the dumbbell-shaped ciliary MEMS vector hydrophone reported by Ji et al. [20], and the hollow cilium cylinder vec- tor hydrophone presented by Yang et al. [21]. However, they increased varying degrees of the difficulty of the hydrophone fabricating process and reduced the consistency of the hydrophone production. And, in reference [21], they did not take into account that the hollow cilium cylinder structure is prone to bubble forming, which has destructive effects on the performance of the hydrophone.

Furthermore, all of the above studies only improved the sensitivity of MEMS hydrophones by changing the cylinder structure or four-beam microstructure alone, without optimizing and improving both. In this paper, through theoretical analysis and derivation, the maximum stress on the beam and the natural frequency of the microstructure are opposite trend changing with each parameter (length, width and height of cantilever, side length of the center block, height and radius of the rigid cylinder) of the microstructure. However, the influence weight of each parameter on the maximum stress and the natural frequency is different. Therefore, the parameters of cylinder structure and fourbeam microstructure are optimized simultaneously, aiming to further improve the sensitivity of the hydrophone and retain the same working bandwidth.

\section{Working Principles}

MEMS bionic vector hydrophone microstructure includes four beams, a rigid cylinder fixed in the middle of the four 


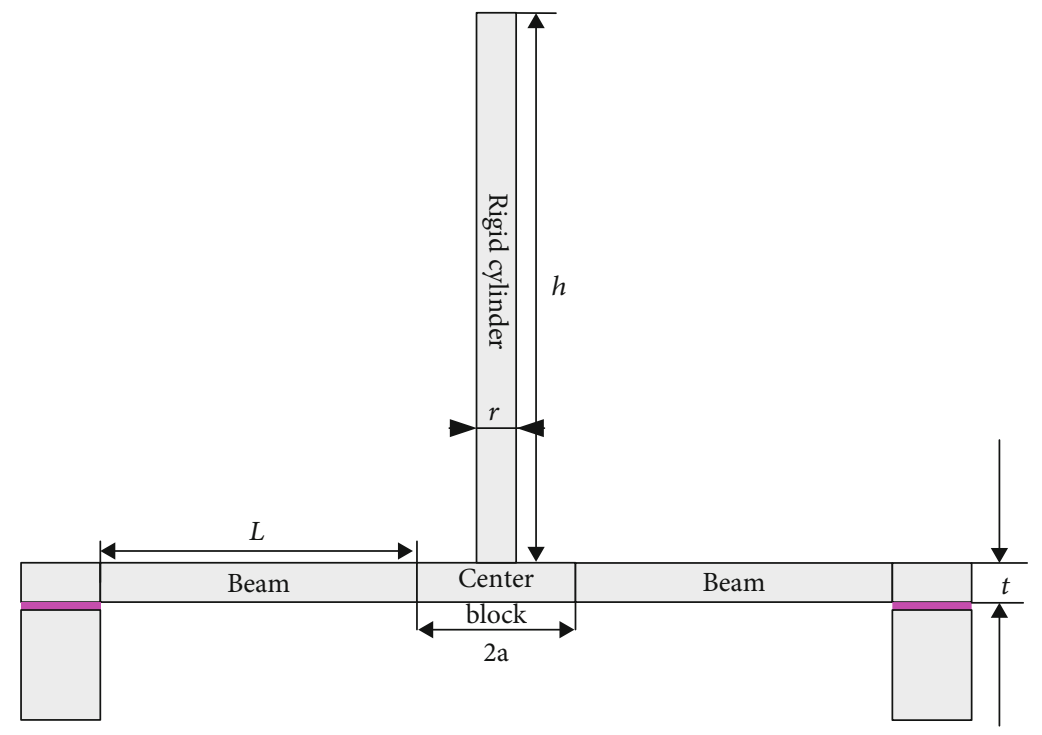

FIgURE 4: The cross-sectional view of the MEMS bionic vector hydrophone microstructure.

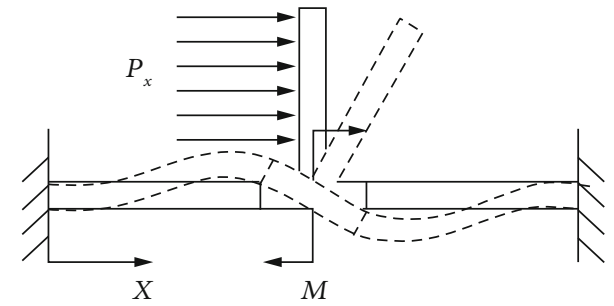

FIGURE 5: Microstructural deformation.

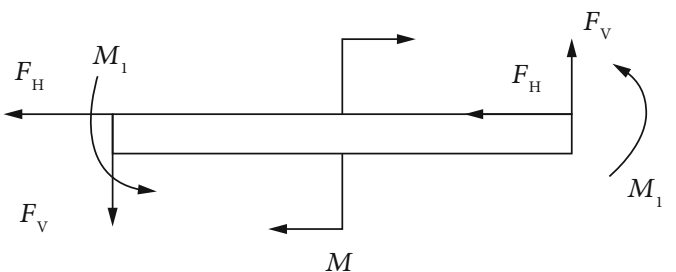

Figure 6: The force analysis diagram of the center block.

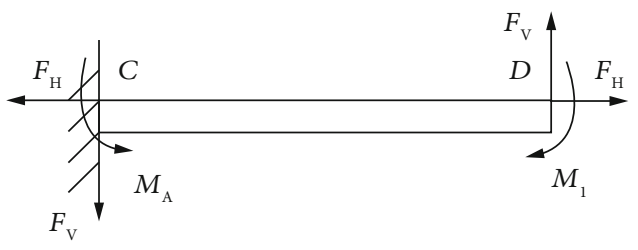

FIgURE 7: Force analysis diagram of a single cantilever beam.

beams, and eight equal strain pizeoresistors in the edge of four beams (as shown in Figure 2) [22]. Figure 2(a) is the three-dimensional model; Figure $2(\mathrm{~b})$ is the twodimensional plan. Eight equal strain pizeoresistors constitute two Wheatstone bridges (as shown in Figure 3).

When sound waves cause vibration of rigid cylinder and make four beams deformed, the stress on four beams will cause the resistance change of the pizeoresistors. Thus, we can get the sound information from the outputs $\left(V_{\text {outx }}\right.$ and $V_{\text {outy }}$ ) of the Wheatstone bridge.

The output voltage of the first Wheatstone bridges can be expressed as

$$
V_{\text {outx }}=\frac{\left(R_{1}+\Delta R_{1}\right)\left(R_{3}+\Delta R_{3}\right)-\left(R_{2}-\Delta R_{2}\right)\left(R_{4}-\Delta R_{4}\right)}{\left(R_{1}+\Delta R_{1}+R_{2}-\Delta R_{2}\right)\left(R_{3}+\Delta R_{3}+R_{4}-\Delta R_{4}\right)} V_{\text {in }} .
$$

When $R_{1}=R_{2}=R_{3}=R_{4}$ and $\Delta R_{1}=\Delta R_{2}=\Delta R_{3}=\Delta R_{4}=\Delta R$,

$$
V_{\text {outx }}=\frac{\Delta R}{R} V_{\text {in }} .
$$

For the P-type strain pizeoresistors [22, 23],

$$
\frac{\Delta R}{R} \approx 71.8 \sigma_{l} \times 10^{-11} .
$$

Therefore, formula (3) can be approximated by:

$$
V_{\text {outx }}=71.8 \times 10^{-11} \times \sigma_{1} \times V_{\text {in }}
$$

where $\sigma_{1}$ is component of longitudinal stress; $V_{\text {in }}$ is the input voltage, and $V_{\text {in }}=10 \mathrm{~V}$. From formula (4), we can conclude that the output $\left(V_{\text {outx }}\right)$ of the Wheatstone bridge is proportional to the stress on the beam.

\section{Optimization Design}

3.1. Microstructure Analysis. The cross-sectional view of the MEMS bionic vector hydrophone microstructure is shown in Figure 4, where $L$ is the length of the beam, $t$ is the thickness of the beam, 2a is the side length of the center block, $h$ is the height of the rigid cylinder, and $r$ is the height of the rigid cylinder. 


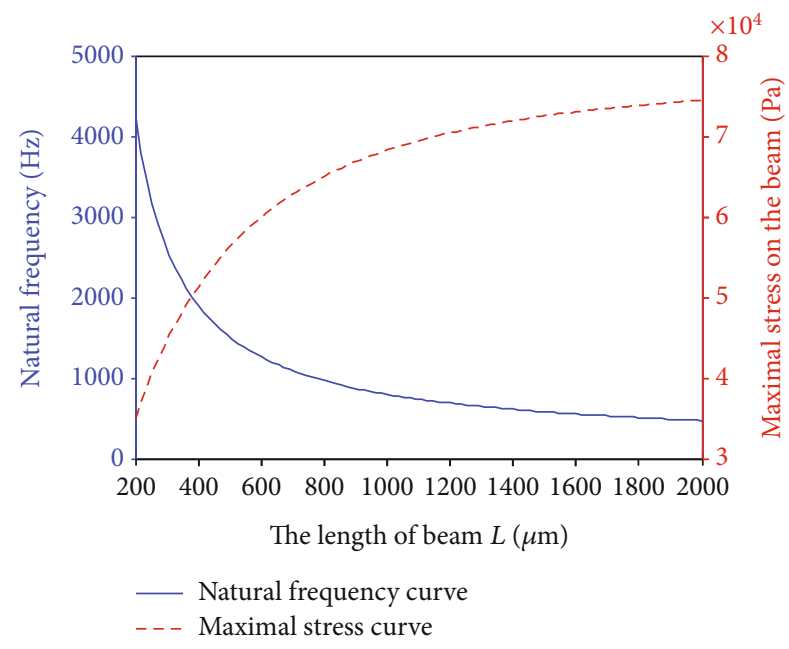

FIGURE 8: Dependence of maximum stress and natural frequency on L.

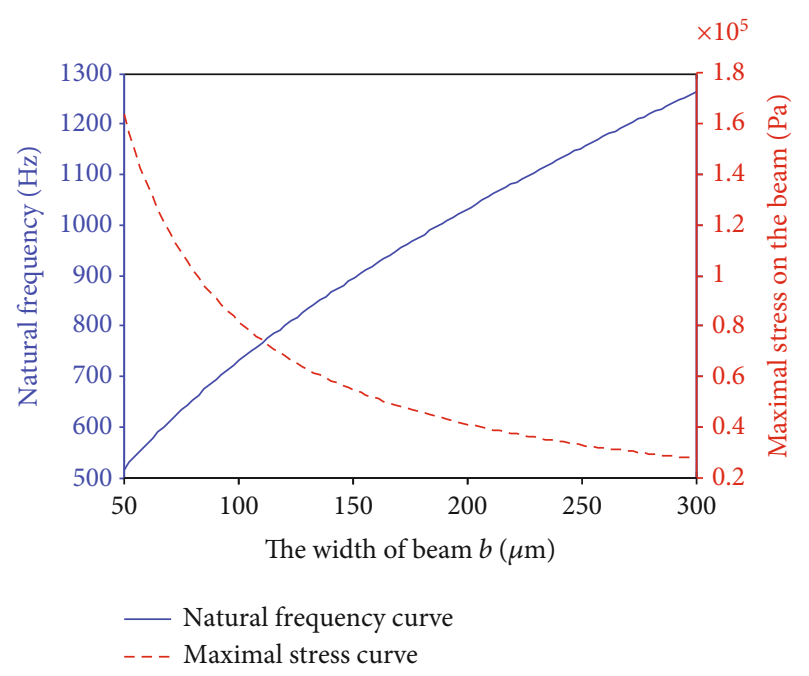

FIGURE 9: Dependence of maximum stress and natural frequency on b.

The mathematical model analysis of the hydrophone microstructure is based on the following assumptions: (a) compared with rigid cylinder, the effective mass of the fourbeam-center block is very small, which can be ignored in the analysis process; (b) the four-beam and the center block is an elastic structure; (c) the rigid cylinder and the frame of the four-beam-center block are rigid. When the rigid cylinder of the hydrophone microstructure is subjected to uniformly distributed loads $P_{x}$ in the $X$ direction. $P_{x}$ will produce two components in the center block of the microstructure, that is, the horizontal force $F_{H}\left(F_{H}=2 P_{x} h r\right)$ along the $X$ direction and the moment $M\left(M=P_{x} h^{2} r\right)$ around the $Y$ direction. Establish the mechanical analysis model of the microstructure (as shown in Figures 5-7). Figure 5 shows deformation when subjected to uniformly distributed loads $P_{x}$. Figure 6 shows the force analysis of the center block, and Figure 7 shows the stress of a single cantilever beam.

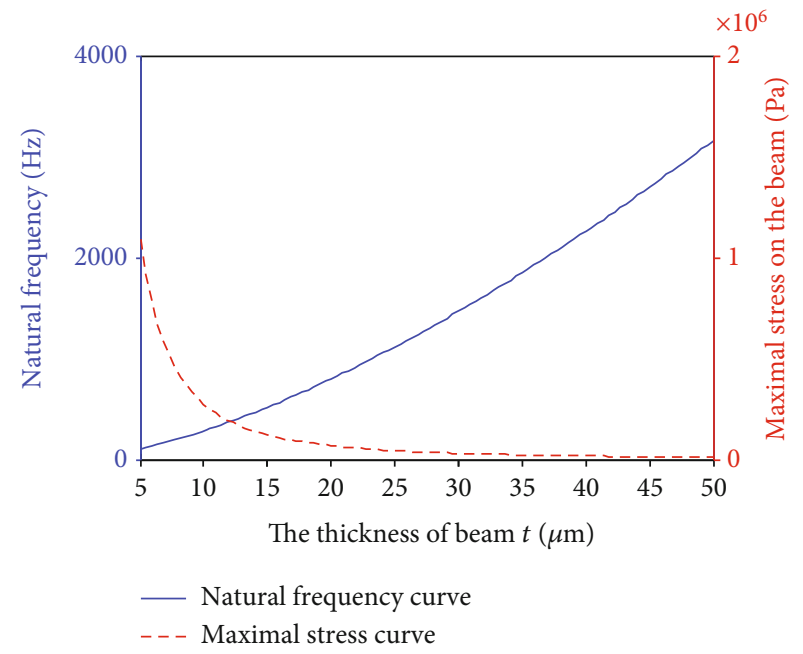

FIGURE 10: Dependence of maximum stress and natural frequency on $t$.

Based on the principles of material mechanics and elastic mechanics [23], for any point $x$ on the cantilever beam, there are:

$$
\sum M=0 \Rightarrow M_{(x)}=-M_{1}+F_{V}(L-x)
$$

The vertical upward force $F_{V}$ at point $D$ can be expressed as:

$$
F_{V}=\frac{3(L+2 a)}{L(2 L+3 a)} M_{1} \text {. }
$$

In Figure 7, the center point of the center block is selected as the research object. Formula (7) can be written as:

$$
\sum M=0 \Rightarrow 2\left(F_{v} \cdot a+M_{1}\right)=M
$$

By substituting formula (6) into (7), it can be obtained that

$$
M_{1}=\frac{L\left(2 L+3_{a}^{2}\right)}{4\left(L^{2}+3 a L+3_{a}^{2}\right)} M .
$$

Substituting formulas (6) and (8) into (5), moment $M_{(x)}$ applied at any point on the beam can be expressed as:

$$
M_{(x)}=\frac{L^{2}+3 a L-3 x(a+L)}{4\left(L^{2}+3 a L+3_{a}^{2}\right)} M
$$




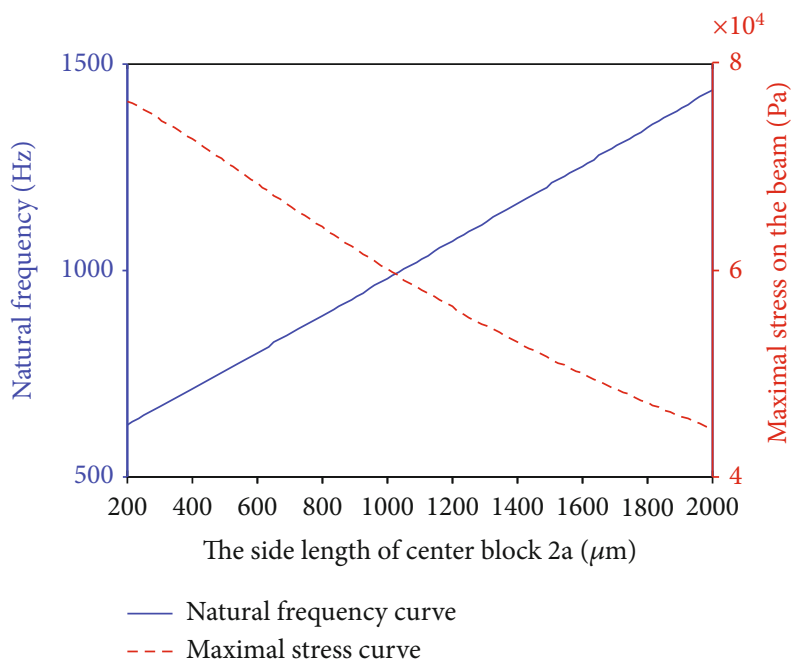

FIGURE 11: Dependence of maximum stress and natural frequency on $2 \mathrm{a}$.

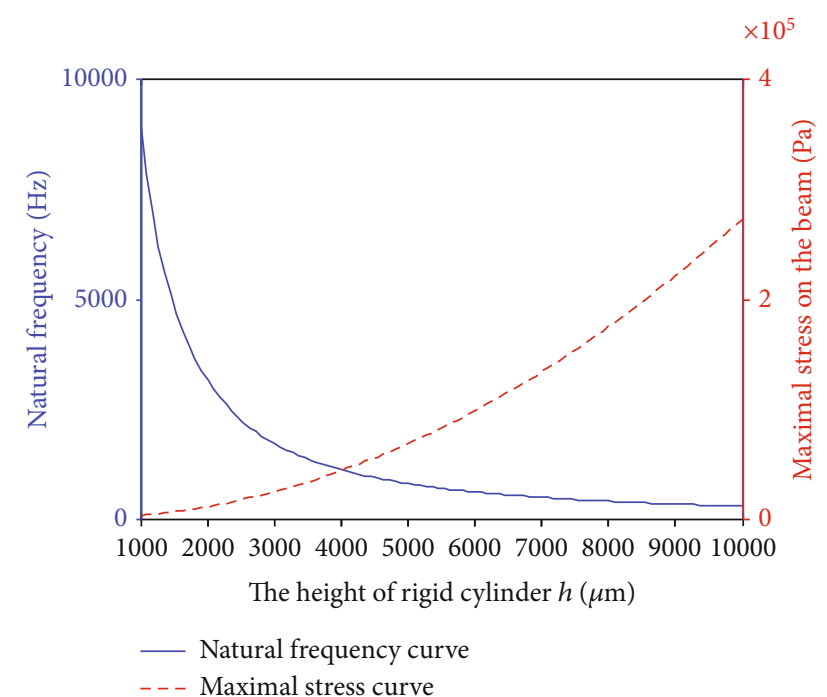

Figure 12: Dependence of maximum stress and natural frequency on $h$.

Therefore, the stress $\sigma_{\left(M_{(x)}\right)}$ of any point $x$ on the cantilever beam under the action of $M_{(x)}$ is:

$$
\sigma_{\left(M_{(x)}\right)}=\frac{M_{(x)}}{W}=\frac{L^{2}+3 a L-3 x(a+L)}{2 / 3 b t^{2}\left(L^{2}+3 a L+3 a^{2}\right)} M,
$$

wherein, $W\left(W=b t^{2 / 6}\right)$ is the bending section modulus of the beam, and $b$ is the width of the beam.

The stress $\sigma_{F_{H}}$ at any point $x$ of a single cantilever beam under the action of $F_{H}$ is:

$$
\sigma_{F_{H}}=\frac{F_{H}}{b t}
$$

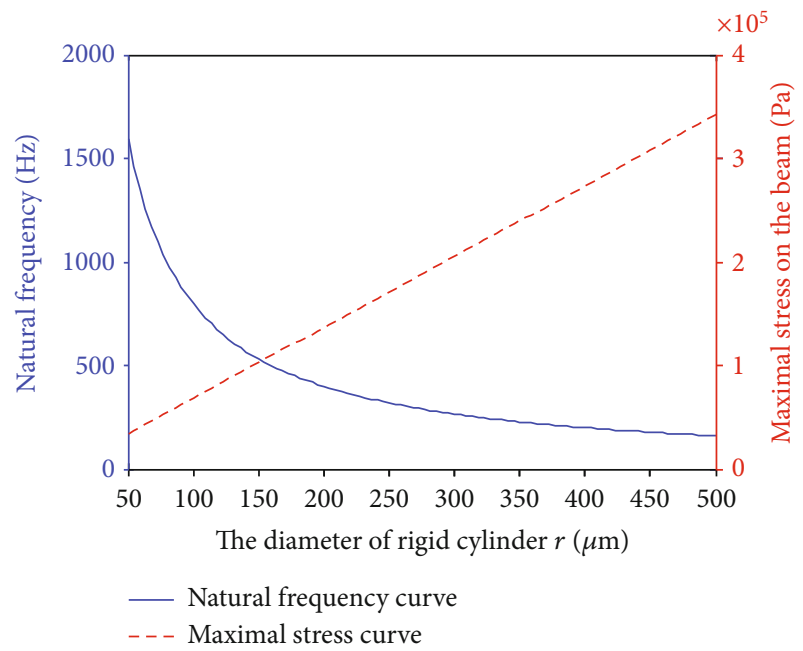

FIGURE 13: Dependence of maximum stress and natural frequency on $r$.

TABLE 1: The geometry parameters of the microstructure.

\begin{tabular}{lcc}
\hline Parameters $(\mu m)$ & 1 & 2 \\
\hline The length of beam $(L)$ & 1000 & 500 \\
The side length of center block $(2 \mathrm{a})$ & 600 & 600 \\
The width of beam $(b)$ & 120 & 80 \\
The thickness of beam $(t)$ & 20 & 12 \\
The thickness of center block $(t)$ & 20 & 12 \\
The height of rigid cylinder $(h)$ & 5000 & 4000 \\
The radius of rigid cylinder $(r)$ & 100 & 100 \\
\hline
\end{tabular}

TABLE 2: Material property parameters.

\begin{tabular}{lccc}
\hline Material & $\begin{array}{c}\text { Density } \rho \\
\left(\mathrm{kg} / \mathrm{m}^{3}\right)\end{array}$ & $\begin{array}{c}\text { Young's modulus } \\
\left(10^{11} \mathrm{~N} / \mathrm{m}^{2}\right)\end{array}$ & $\begin{array}{c}\text { Poisson's } \\
\text { ratio }\end{array}$ \\
\hline $\begin{array}{l}\text { Si } \\
\begin{array}{l}\text { Rigid } \\
\text { cylinder }\end{array}\end{array}$ & 2330 & 1.65 & 0.278 \\
\hline
\end{tabular}

Thus, the stress $\sigma_{(x)}$ at any point $x$ of a single cantilever beam under the combined action of $M_{(x)}$ and $F_{H}$ is

$$
\sigma_{(x)}= \pm \frac{L^{2}+3 a L-3 x(a+L)}{(2 / 3) b t^{2}\left(L^{2}+3 a L+3 a^{2}\right)} M \pm \frac{F_{H}}{b t}
$$

It can be known from formula (12) that the maximal stress on the beam is on the both ends. That is, $\sigma_{(0)}$ is maximal (as shown in formula (13)).

$$
\sigma(0)= \pm \frac{L^{2}+3 a L}{(2 / 3) b t^{2}\left(L^{2}+3 a L+3 a^{2}\right)} M \pm \frac{F_{H}}{b t}
$$




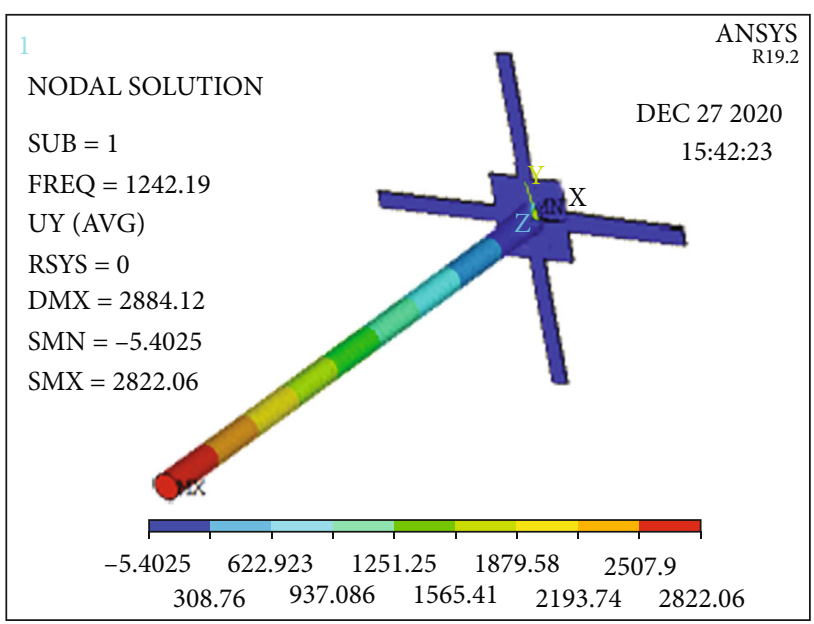

(a) Previous microstructure

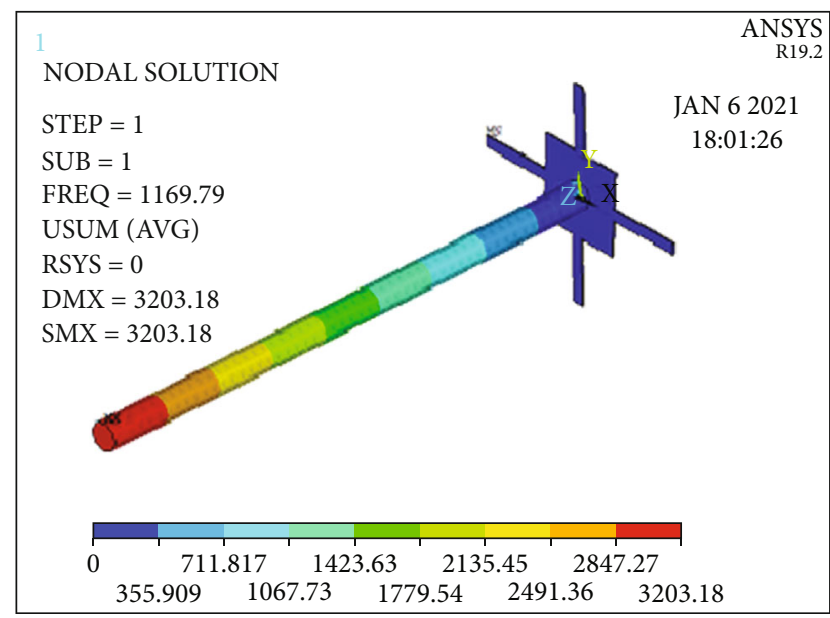

(b) Optimized microstructure

FIgURE 14: The first order modal analysis.

According to material mechanics theory, the stiffness of the microstructure on the $X$ or $Y$ direction can be calculated which can be expressed as $[23,24]$ :

$$
K_{x}=K_{y}=\frac{2 E b t^{3}}{L h^{2}}\left(\frac{a^{2}}{L^{2}}+\frac{a}{L}+\frac{1}{3}\right),
$$

where $E$ is the Young's modulus of silicon material.

The nature frequency of the microstructure is can be expressed as:

$$
f=\frac{1}{2 \pi} \sqrt{\frac{K}{m}}=\frac{1}{2 \pi} \sqrt{\frac{2 E b t^{3}}{m L h^{2}}\left(\frac{a^{2}}{L^{2}}+\frac{a}{L}+\frac{1}{3}\right)},
$$

where $m$ is the mass of the rigid cylinder. $K$ is the stiffness of the microstructure and $K=K_{x}=K_{y}$.

From formulas (14) and (15), it can be concluded that the natural frequency of the microstructure and the maximum stress on the cantilever beam vary with the microstructural parameters (length, width and height of cantilever, side length of the center block, height and radius of the rigid cylinder) (as shown in Figures 8-13).

From Figures $8-13$, it can be seen that the microstructural parameters have different weight effects on the natural frequency of the microstructure and the maximum stress on the beam. And, $2 \mathrm{a}$ and $L$ have almost the same weight effect on the maximum stress and the natural frequency; $t$ and $b$ have a greater weight effect on the maximum stress than the natural frequency; $r$ and $h$ have a greater weight effect on the natural frequency than the maximum stress. Therefore, MEMS vector hydrophone microstructural parameters can be designed reasonably to obtain the maximum stress, and the natural frequency hardly changes.
3.2. Optimization Design. The optimal solution is any feasible solution that makes the objective function obtain the optimal value (maximum or minimum value) in the feasible region.

The maximum stress $\sigma_{(0)}$ and nature frequency $f$, respectively, can be seen as the multivariate functions of the microstructural parameters $(L, b, t, a, h$, and $r$ ) (as shown in formulas (16) and (17)).

$$
\begin{aligned}
& \sigma(L, b, t, a, h, r)=\frac{L^{2}+3 a L}{(2 / 3) b t^{2}\left(L^{2}+3 a L+3 a^{2}\right)} M+\frac{F_{H}}{b t} \\
& f(L, b, t, a, h, r)=\frac{1}{2 \pi} \sqrt{\frac{2 E b t^{3}}{m L h^{2}}\left(\frac{a^{2}}{L^{2}}+\frac{a}{L}+\frac{1}{3}\right)} .
\end{aligned}
$$

The first set of values $\left(L_{1}, b_{1}, t_{1}, a_{1}, h_{1}, r_{1}\right)$ as shown in Table 1 is commonly used as the microstructure parameters. According to the actual processing conditions and requirements, the ranges of the variable parameters are set as follows: (1) $500 \leq L \leq 1500$, (2) $80 \leq b \leq 160$, (3) 8 $\leq t \leq 40$, (4) $200 \leq a \leq 300$, (5) $2000 \leq h \leq 10000$, (6) 100 $\leq r \leq 200$, and (7) $f\left(L_{1}, b_{1}, t_{1}, a_{1}, h_{1}, r_{1}\right) \leq f(L, b, t, a, h, r)$. $D(L, b, t, a, h, r)$ is regarded as the feasible zone constituted by the above seven constraint conditions, and $\sigma(L$, $b, t, a, h, r)$ is viewed as the objective function. Searching the maximum of $\sigma(L, b, t, a, h, r)$ in $D(L, b, t, a, h, r)$ by MATLAB, the optimal solution $\left(L_{2}, b_{2}, t_{2}, a_{2}, h_{2}, r_{2}\right)$ is obtained when $\sigma(L, b, t, a, h, r)$ acquire the max (as shown in the second set of values of Table 1$)$.

\section{Simulation Analysis and Fabrication}

4.1. Simulation Analysis. The finite element models of the previous microstructure and the optimized microstructure have been, respectively, established by ANSYS software. The material property parameters are shown in Table 2. The modal analysis and static analysis have been carried 


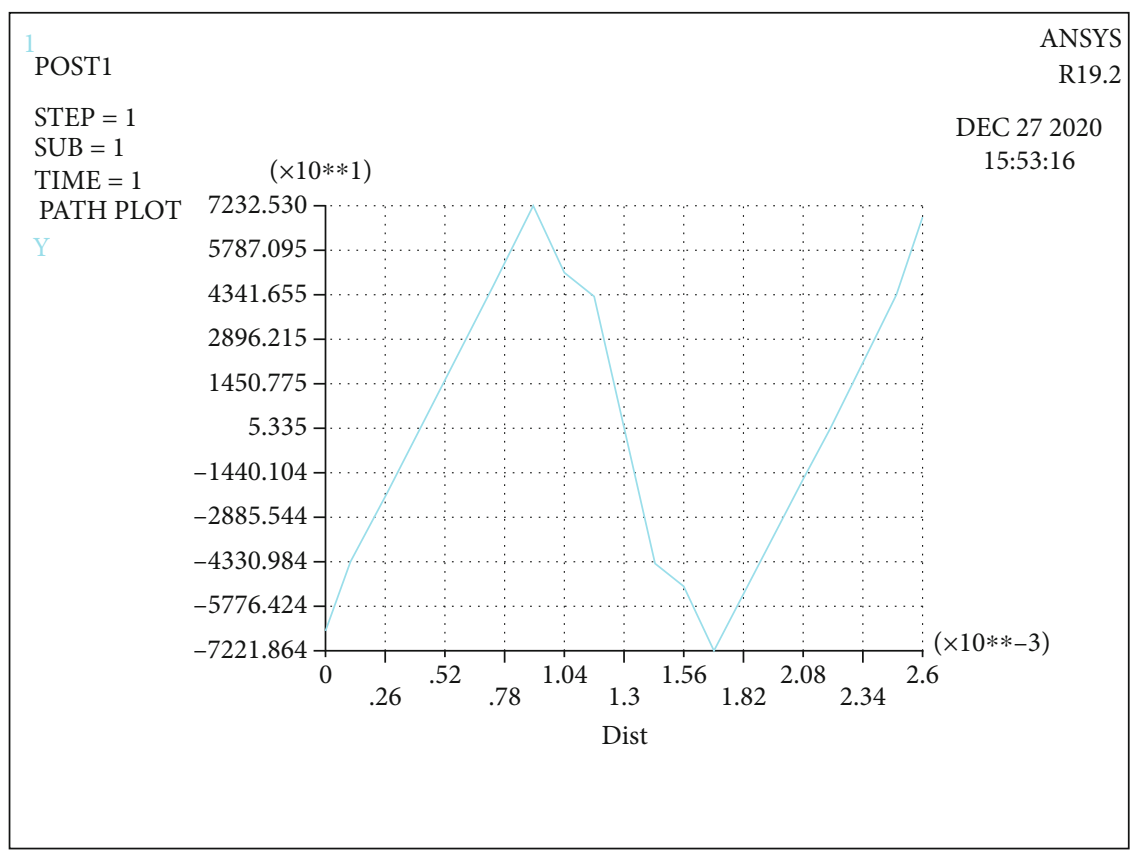

(a) Previous microstructure

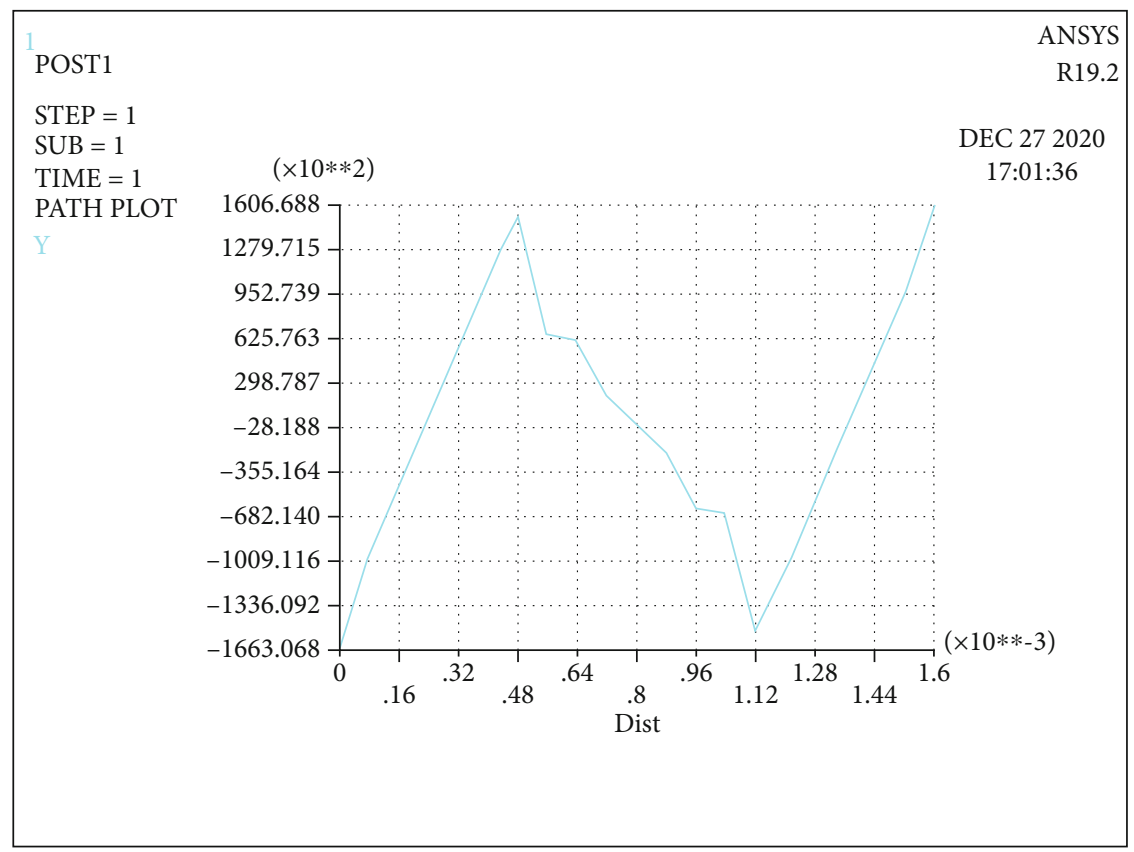

(b) Optimized microstructure

FIGURE 15: Stress analysis.

out. The nature frequency of the microstructures can be obtained by modal diagrams (as shown in Figure 14). And the stress distribution curves on the beams are shown in Figure 15.

It can be seen from Figures 14 and 15 that the nature frequency of the optimized microstructure is $1170 \mathrm{~Hz}$, which is close to that $(1242 \mathrm{~Hz})$ of previous microstructure, and the maximum stress on the beam of the optimized microstructure is $160669 \mathrm{~Pa}$, which is more than twice as much as that $(72325 \mathrm{~Pa})$ of previous microstruc- ture. That is to say, the sensitivity of the optimized microstructure has been doubled, and the nature frequency almost remains unchanged. Thus, the optimization design of the microstructure is feasible.

4.2. Fabrication. The four-beam microstructure is fabricated by standard MEMS technology which mainly includes photolithography, oxidation, etching, ion implantation, and sputtering $[25,26]$. The main process is shown in Figure 16. (a) An N-type 4-inch SOI (12 $\mu \mathrm{m}$ device layer, 


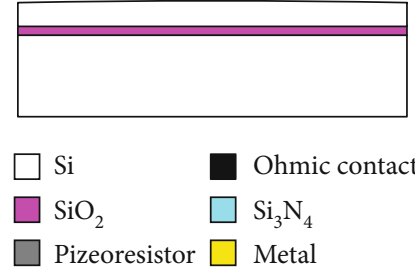

(a) SOI

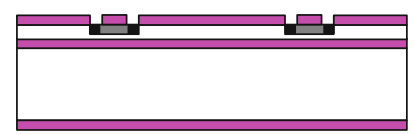

$\square \mathrm{Si}$ Ohmic contact

$\mathrm{SiO}_{2} \quad \square \mathrm{Si}_{3} \mathrm{~N}_{4}$

Pizeoresistor $\square$ Metal

(c) Ohmic contact

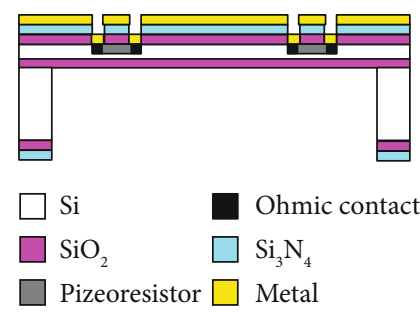

(e) Metal wire

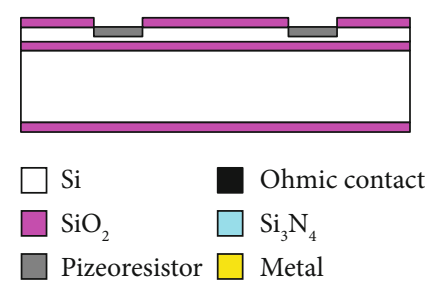

(b) Pizeoresistor

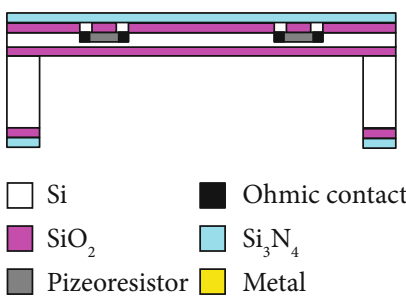

(d) Etching back substrate

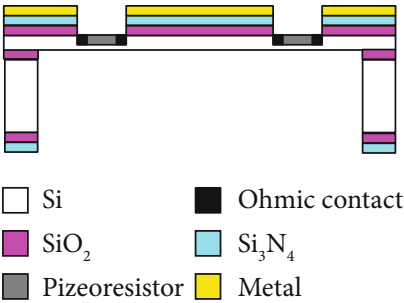

(f) Four-beam structure

FIGURE 16: The processing technology of the optimized four-beam microstructure.

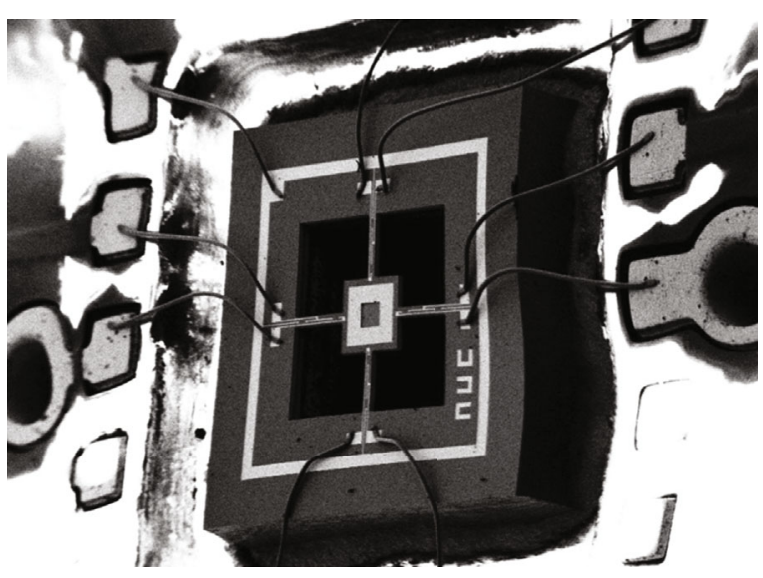

FIGURE 17: SEM diagram of MEMS four-beam microstructure.

$2 \mu \mathrm{m}$ buried oxide layer, and $400 \mu \mathrm{m}$ substrate layer) with crystal plane (100) and crystal orientation $<100>$ is prepared. (b) The SOI is oxidized at $950^{\circ} \mathrm{C}$ to form $1000 \AA \mathrm{SiO}_{2}$ on both sides, and the front $\mathrm{SiO}_{2}$ is etched by ICP to form the piezoresistor windows. The boron ions with $100 \mathrm{keV}$ energy and $4 \times 10^{18} \mathrm{~cm}^{-3}$ surface concentration are implanted, and then anneal to form the piezoresistors. (c) The SOI is reoxidized, and the front $\mathrm{SiO}_{2}$ is etched by ICP to form ohmic contact region. The denser boron ions with $100 \mathrm{keV}$ energy and $4 \times$ $10^{21} \mathrm{~cm}^{-3}$ surface concentration are implanted, and then, the ohmic contacts are formed by annealing. (d) $1100 \AA$ $\mathrm{Si}_{3} \mathrm{~N}_{4}$ is deposited on both sides of the SOI by PECVD. The
$\mathrm{Si}_{3} \mathrm{~N}_{4}$ and $\mathrm{SiO}_{2}$ on the back side of SOI are etched by RIE, and then, etch the backside silicon using $\mathrm{KOH}$ and self-stop at the $\mathrm{SiO}_{2}$ layer. (e) $500 \AA \mathrm{Cr}$ and $1000 \AA \mathrm{Au}$ are deposited on the front side and etched by iodine water to realize the connection of the Wheatstone bridge. (f) $\mathrm{Si}_{3} \mathrm{~N}_{4}$ and $\mathrm{SiO}_{2}$ on the front side are etched by RIE, and $\mathrm{SiO}_{2}$ on the back side is etched by RIE, and then, the cantilever structure is released.

The four-beam microstructure (MEMS chip) is fabricated as shown in Figure 17. The high aspect ratio microstructure cannot be processed through the current domestic MEMS processing lines, so it is necessary to bond the fourbeam microstructure and the rigid cylinder. Then, the two hydrophones (previous one and optimized one) have been fabricated (as shown in Figure 18).

\section{Performance Test}

The calibration tests for these two hydrophones without package have been, respectively, performed in the standing wave barrel with the method of comparison [27]. During testing, one of these two hydrophones (tested hydrophone) and a standard hydrophone are placed in the standing wave barrel filled with silicone oil. The standard hydrophone is the RS-100 piezoelectric sound pressure hydrophone with sensitivity of $-180 \mathrm{~dB}(0 \mathrm{~dB}$ ref $1 \mathrm{~V} / \mu \mathrm{Pa})$ and bandwidth of $20 \mathrm{~Hz} \sim 100 \mathrm{kHz}$ used as a reference hydrophone for the calibration test. The sensitivity of the tested hydrophone at different frequency can be acquired by comparing the output voltage values of the two hydrophones (tested hydrophone 


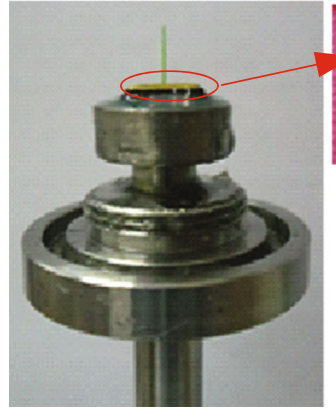

Previous structure

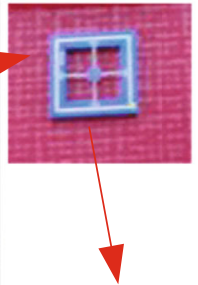

Previous chip

FIGURE 18: The diagram of two hydrophones without package.

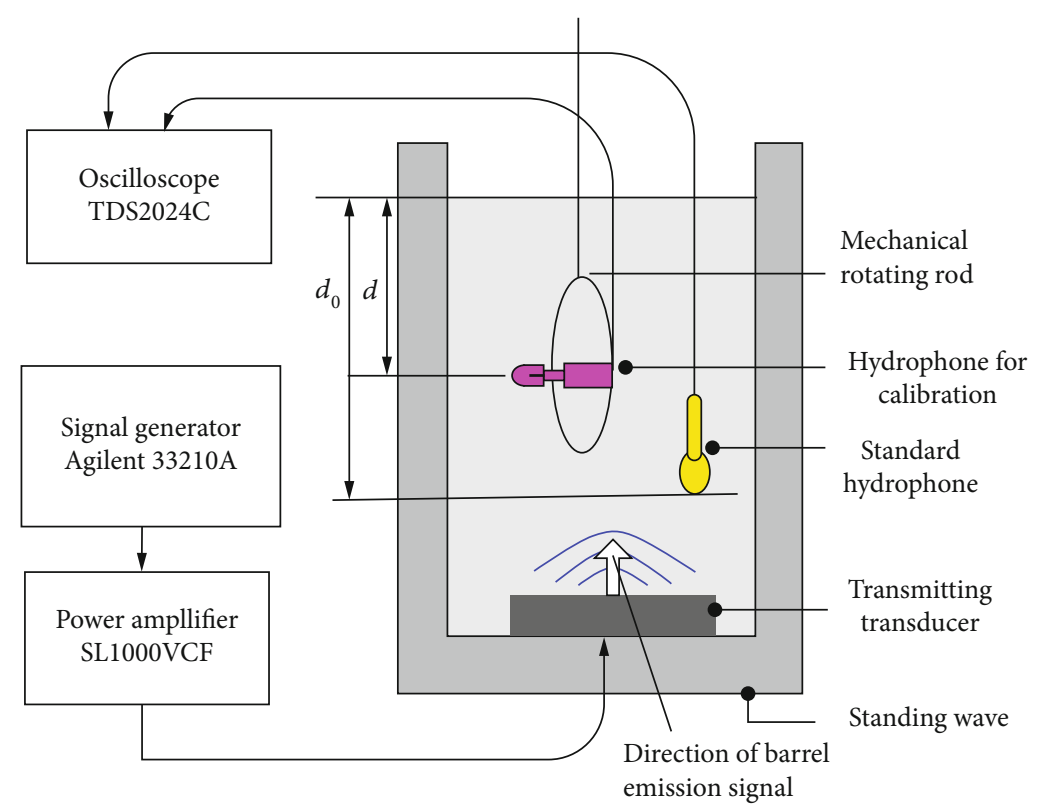

FIGURE 19: Schematic diagram of the calibration device.

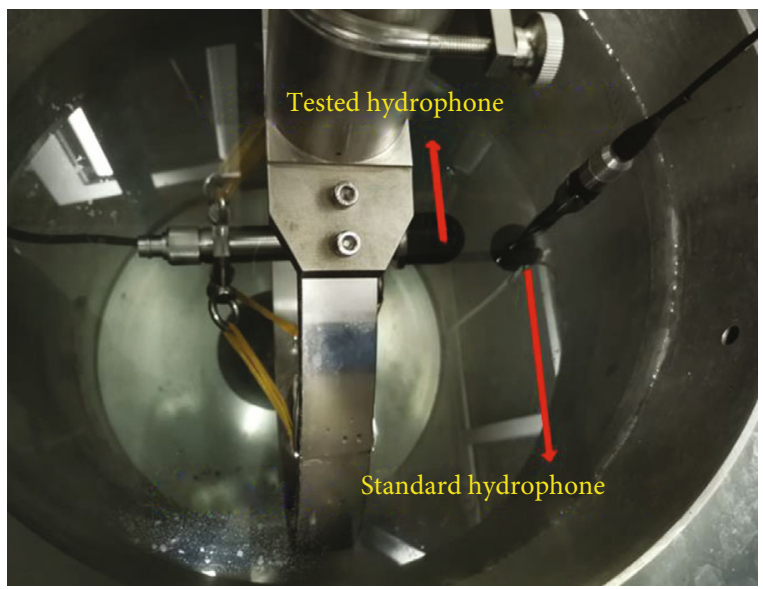

Figure 20: The calibration system.

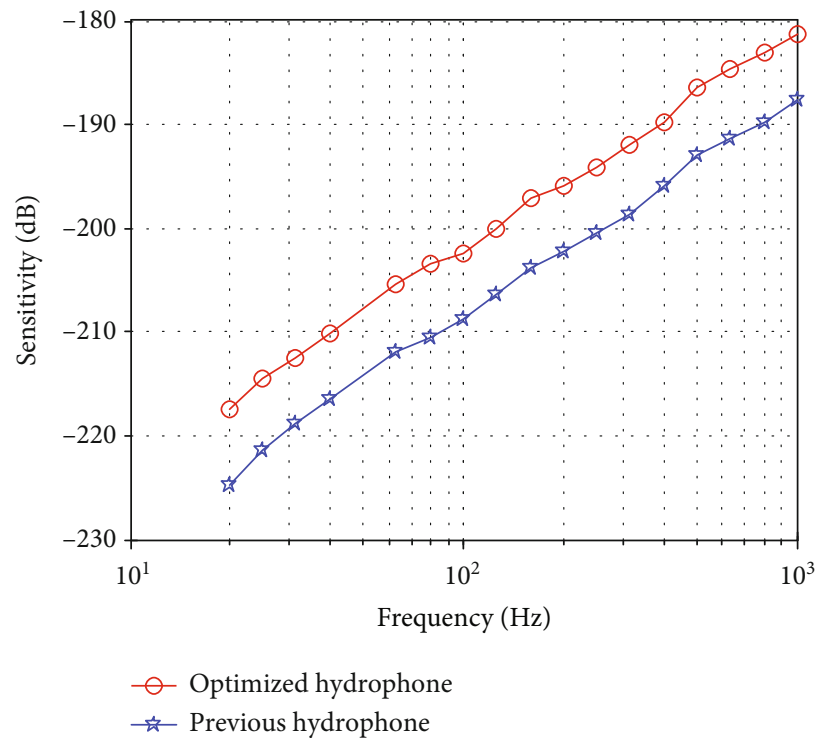

Figure 21: The sensitivity curves in $X$ direction. 


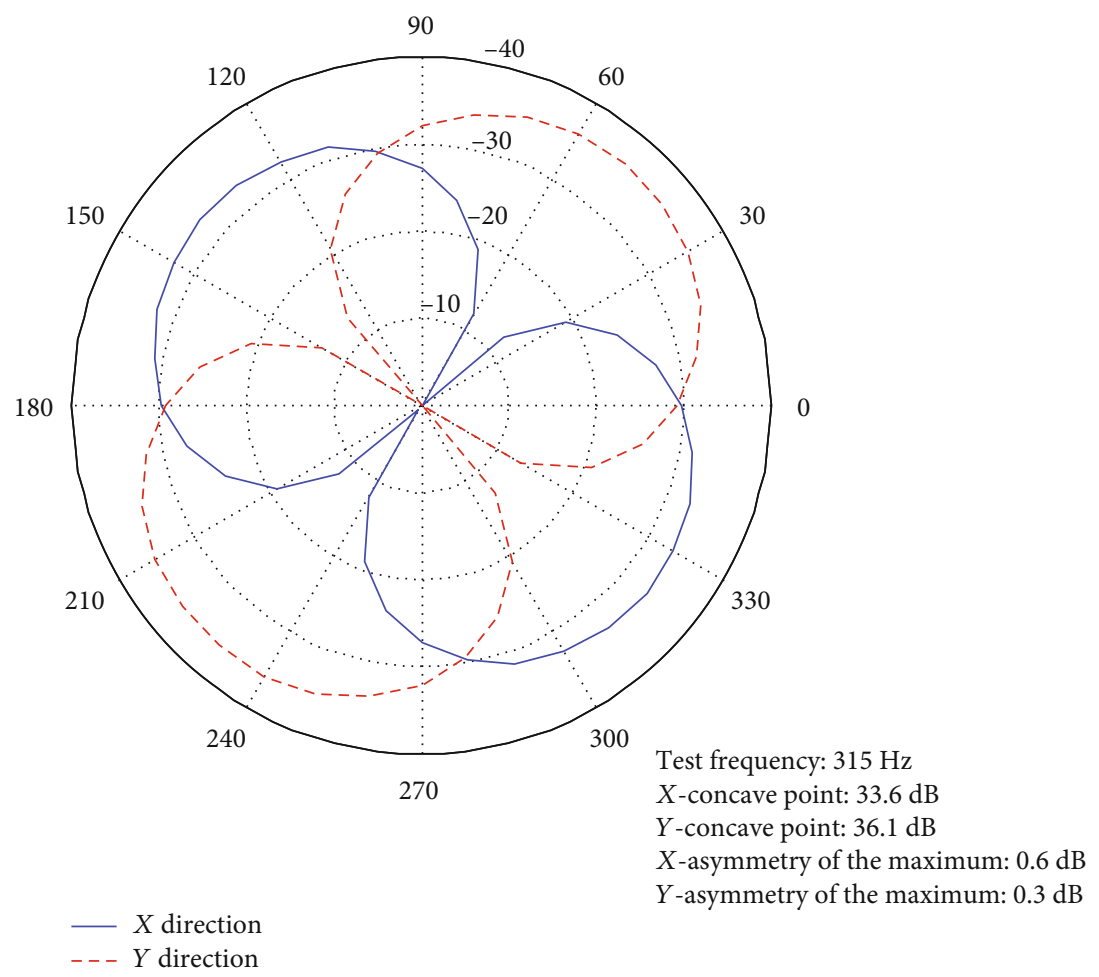

Figure 22: Directivity pattern at a frequency of $315 \mathrm{~Hz}$.

and standard hydrophone). The schematic diagram of the calibration devices is shown in Figure 19, and the calibration system is shown in Figure 20. During the testing, data are recorded in one-third-octave frequency points from $20 \mathrm{~Hz}$ to $1 \mathrm{kHz}$. When the sensitivity of $X$ or $Y$ direction is calibrated, it must be made sure that the acoustic wave propagation direction of the standing wave barrel is paralleled to the corresponding maximum sensitivity direction.

The $X$ direction is as an example in this paper. The sensitivity $M_{x}$ of the tested hydrophone can be expressed as [27]:

$$
M_{x}=M_{0} \frac{e_{x}}{e_{0}} \bullet \frac{\sin k d_{0}}{\cos k d},
$$

where $M_{0}$ is the voltage sensitivity of the standard hydrophone; $e_{x}$ and $e_{0}$ are the open output voltage of the tested hydrophone and the standard hydrophone, respectively; $d$ is the depth of the tested hydrophone; $d_{0}$ is the depth of the standard hydrophone; $k$ is wave number. The sensitivity testing results of these two hydrophones are shown in Figure 21.

Then, the directivity pattern of the optimized hydrophone is measured by the same equipment as shown in Figure 20. At the tested frequency, rotate the optimized hydrophone from $0^{\circ}$ to $360^{\circ}$ and record the hydrophone's outputs $\left(e_{\theta}\right)$ at each degree. The recorded data should be normalized by formula (19).

$$
L=20 \log \left(\frac{e_{\theta}}{e_{\max }}\right)
$$

where $e_{\max }$ is the maximum of the outputs. The directivity pattern is obtained by plotting the normalized data $(L)$ in polar coordinates. The directivity pattern at $315 \mathrm{~Hz}$ is depicted in Figure 22.

From Figure 21, it can be obtained that the sensitivity of the optimized hydrophone is $-181.2 \mathrm{~dB} @ 1 \mathrm{kHz}(0 \mathrm{~dB}$ reference $1 \mathrm{~V} / \mu \mathrm{Pa}$ ), which is about $6.5 \mathrm{~dB}$ higher than that of the previous hydrophone, and they all range in working bandwidth from $20 \mathrm{~Hz}$ to $1000 \mathrm{~Hz}$. Figure 22 shows that the optimized hydrophone has a good directional pattern in the form of an " 8 " shape and excellent symmetry property. Therefore, the optimized microstructure can improve the sensitivity of the hydrophone and maintain the same working bandwidth with the previous microstructure.

\section{Conclusion}

To better realize the engineering application of the MEMS vector hydrophone, this paper introduces the optimal designs of the hydrophone microstructure to further improve its sensitivity without changing working bandwidth. The mechanics analysis of the microstructure is carried out, and the objective function and feasible region are established to calculate the optimal solution of the microstructure. And the optimal designs have been proved through ANSYS simulation.

Then, the optimized microstructure has been fabricated by the standard MEMS technology. Finally, the two hydrophones (previous one and optimized one) have been fabricated and tested in standing wave barrel to verify the feasibility of the optimization. The test results show that the 
receiving sensitivity of the optimized hydrophone reaches up to $-181.2 \mathrm{~dB} @ 1 \mathrm{kHz}(0 \mathrm{~dB}$ reference $1 \mathrm{~V} / \mu \mathrm{Pa})$, improved $6.5 \mathrm{~dB}$, and whose working bandwidth is $20-1000 \mathrm{~Hz}$ without changed. Moreover, the optimized hydrophone has superior " 8 " shape directivity and concave point depth. Thus, the performance of the optimized hydrophone has been greatly improved compared with that of the previous hydrophone. The optimization provides new ideas to improve the performance of MEMS bionic vector hydrophone and lays the further foundation for its engineering application.

\section{Data Availability}

The data used to support the findings of this study are available from the corresponding author upon request.

\section{Conflicts of Interest}

The authors declare that there is no conflict of interest regarding the publication of this paper.

\section{Acknowledgments}

This work was funded by the National Natural Science Foundation of China (Grant nos. 51805154 and 51975191) and Scientific Research Foundation of Hubei University of Technology (GCRC2020010).

\section{References}

[1] S. L. Fan, Thinking of China's Sea Power and Ocean Strategy, [Ph.D. thesis], Central Party School, 2007.

[2] D. H. Liu, C. C. Lian, F. M. Liu, C. J. Wang, R. X. Ji, and X. X. $\mathrm{Li}$, "Some views on ocean strategic layout of the Atlantic Ocean for China," Ocean Development and Management, vol. 33, no. 5, pp. 3-7, 2016.

[3] S. T. Yang, The Research of Deep-Water Vector Hydrophone, [Ph.D. thesis], Harbin Institute of Technology, 2010.

[4] K. Kim, Investigation of an Underwater Acoustic Vector Sensor, [Ph.D. thesis], The Pennsylvania State University, 2002.

[5] G. P. Zhang, C. Zheng, and W. S. Lin, "Steering acoustic intensity estimator using a single acoustic vector hydrophone," Journal of Sensors, vol. 2018, Article ID 8526092, 10 pages, 2018.

[6] M. Liu, L. Nie, G. Zhang, W. Zhang, and J. Zou, "Realization of a composite MEMS hydrophone without left-right ambiguity," Sensors and Actuators A: Physical, vol. 272, pp. 231-241, 2018.

[7] P. Amiri, Z. Kordrostami, and K. Hassanli, "Design of a MEMS bionic vector hydrophone with piezo-gated MOSFET readout," Microelectronics Journal, vol. 98, article 104748, p. 7, 2020.

[8] A. Hossain and A. Mian, "Four-terminal square piezoresistive sensors for MEMS pressure sensing," Journal of Sensors, vol. 2017, Article ID 6954875, 11 pages, 2017.

[9] J. Kim, S. Pyo, and Y. Roh, "Analysis of a thickness-shear mode vibrator for the accelerometer in vector hydrophones," Sensors and Actuators, A: Physical, vol. 266, pp. 9-14, 2017.

[10] B. A. Ganji, M. S. Nateri, and M. Dardel, "Design and modeling of a novel high sensitive MEMS piezoelectric vector hydrophone," Microsystem Technologies, vol. 24, no. 4, pp. 20852095, 2018.
[11] C. Xue, S. Chen, W. Zhang, B. Zhang, G. Zhang, and H. Qiao, "Design, fabrication, and preliminary characterization of a novel mems bionic vector hydrophone," Microelectronics Journal, vol. 38, no. 10-11, pp. 1021-1026, 2007.

[12] C. Xue, Z. Tong, B. Zhang, and W. Zhang, "A novel vector hydrophone based on the piezoresistive effect of resonant tunneling diode," IEEE Sensors Journal, vol. 8, no. 4, pp. 401$402,2008$.

[13] M. Liu, G. Zhang, X. Song, Y. Liu, and W. Zhang, "Design of the monolithic integrated array MEMS hydrophone," IEEE Sensors Journal, vol. 16, no. 4, pp. 989-995, 2016.

[14] Q. Xu, G. Zhang, Y. Zhao et al., "New insight into contradictive relationship between sensitivity and working bandwidth of cilium MEMS bionic vector hydrophone," Journal of Micromechanics and Microengineering, vol. 29, no. 11, article 115016, 2019.

[15] Y. Liu, R. Wang, G. Zhang et al., “"Lollipop-shaped” highsensitivity microelectromechanical systems vector hydrophone based on parylene encapsulation," Journal of Applied Physics, vol. 118, no. 4, article 044501.1, 2015.

[16] G. Zhang, J. Ding, W. Xu et al., "Design and optimization of stress centralized MEMS vector hydrophone with high sensitivity at low frequency," Mechanical Systems and Signal Processing, vol. 104, pp. 607-618, 2018.

[17] W. Xu, Y. Liu, G. Zhang et al., "Development of cup-shaped microelectromechanical systems-based vector hydrophone," Journal of Applied Physics, vol. 120, no. 12, article 124502, 2016.

[18] Q. Xu, G. Zhang, J. Ding et al., "Design and implementation of two-component cilia cylinder MEMS vector hydrophone," Sensors and Actuators, A: Physical, vol. 277, pp. 142-149, 2018.

[19] L. Zhang, Q. Xu, G. Zhang et al., "Design and fabrication of a multipurpose cilia cluster MEMS vector hydrophone," Sensors and Actuators, A: Physical, vol. 296, pp. 331-339, 2019.

[20] S. Ji, L. Zhang, W. Zhang et al., "Design and realization of dumbbell-shaped ciliary MEMS vector hydrophone," Sensors and Actuators, A: Physical, vol. 311, p. 9, 2020.

[21] X. Yang, Q. Xu, G. Zhang et al., "Design and implementation of hollow cilium cylinder MEMS vector hydrophone," Measurement, vol. 168, article 108309, 2021.

[22] K. V. Meena, R. Mathew, J. Leelavathi, and A. R. Sankar, "Performance comparison of a single element piezoresistor with a half-active Wheatstone bridge for miniaturized pressure sensors," Measurement, vol. 111, pp. 340-350, 2017.

[23] S. Chen, Research of MEMS Bionic Vector Hydrophone Based on Silicon, [Ph.D. thesis], The North University of China, 2008.

[24] Z. M. Jian, G. J. Zhang, M. R. Liu, and W. D. Zhang, "Microstructure optimization design of the MEMS bionic vector hydrophone," Micronanoelectronic Technology, vol. 51, no. 9, pp. 576-582, 2014.

[25] H. Guo, X. Li, Q. Zhu et al., "Imaging nano-defects of metal waveguides using the microwave cavity interference enhancement method," Nanotechnology, vol. 31, no. 45, article 455203, 2020.

[26] M. Liu, L. Nie, S. Li et al., "Array MEMS vector hydrophone oriented at different direction angles," Sensors, vol. 19, no. 19, article 4282, 2019.

[27] J. X. Fan, The Study of Vector Hydrophone Calibration Equipment, [M.S. thesis], Harbin Engineering University, 2007. 